\title{
Erratum: Fermi-Surface Anisotropy in Potassium and the Effect of Hydrostatic Pressure*
}

\author{
I. M. Templeton \\ Division of Physics, National Research Council of Canada, Ottawa, Canada
}

(Received October 28, 1981)

The radial pressure coefficient anisotropy was determined incorrectly, in that an extra factor of 0.5 was used in the inversion. The following corrections should be made:

Table VIII: Terms $2-6$ for $d \ln r / d p$ should be doubled. They should now read $0.01940,-0.01477,0.00912,0.00276$, and -0.00159 .

Fig. 14: The values at $\langle 100\rangle,\langle 110\rangle$, and $\langle 111\rangle$ should be $8.74,8.70$, and 8.42 , respectively. Units are $10^{-3} / \mathrm{kbar}$ : the solid line corresponds to $8.6 \times 10^{-3} / \mathrm{kbar}$, and the contour interval is $0.04 \times 10^{-3} / \mathrm{kbar}$.

Fig. 16: The values at $\langle 100\rangle,\langle 110\rangle$, and $\langle 111\rangle$ should be $+17,+12$, and -21 , respectively. The contour interval is four units.

The footnote to Section 4.2 refers to area pressure coefficient anisotropies in $\mathrm{Rb}$ and $\mathrm{Cs}$. 